\title{
VLBI Imaging of Luminous Infrared Galaxies: Starbursts \& AGN
}

\author{
Harding E. Smith ${ }^{1}$ \\ Center for Astrophysics 83 Space Sciences and Department of Physics, \\ University of California, San Diego, La Jolla, CA 92093-0424, USA
}

Carol J. Lonsdale

Infrared Processing and Analysis Center, California Institute of Technology, Pasadena, CA 91125, USA

Colin J. Lonsdale

Haystack Observatory, Massachusetts Institute of Technology, Westford, MA 01886, USA

Philip J. Diamond

National Radio Astronomy Observatory, Socorro, NM 87801, USA

\begin{abstract}
.
Luminous Infrared Galaxies (LIGs) are locally more numerous than normal galaxies, AGN, and QSOs above $L \sim 10^{11} L_{\odot}$ and may be the evolutionary precursors of classical radio-quiet quasars. VLBI observations of a complete sample show that high- $T_{b}$ radio cores are common, perhaps universal among LIGs. VLBI imaging shows that these radio cores may be produced by intense starbursts which generate luminous radio supernovae, as in the case of Arp 220 (Smith et al. 1998), or by a classical AGN core, as in the case of Mrk 231, which we interpret as a newly formed QSO emerging from a starburst. Compact $\mathrm{OH} 1667 \mathrm{MHz}$ maser emission appears to be common in LIGs and may be related to AGN activity. These results lend further support to the scenario suggested by Sanders et al (1988) in which mergers of gas-rich galaxies lead first to luminous starbursts which evolve into radio-quiet quasars.
\end{abstract}

\section{Luminous Infrared Galaxies}

The most luminous galaxies in the Local Universe are Luminous Infrared Galaxies (LIGs) which emit the vast majority of their radiant power in the far-infrared between about $40-120 \mu \mathrm{m}$. These are gas-rich systems which are in the late stages of collisions or mergers and extrapolation from the properties of lower luminosity starburst galaxies suggests that the LIGs should be active star-forming systems

\footnotetext{
${ }^{1}$ also, Infrared Processing and Analysis Center, Caltech/JPL, Pasadena, CA 91125
} 
(see Sanders \& Mirabel 1996 for a review). The LIGs also show many characterisatics of AGN and their luminosities reach values comparable to those of luminous QSOs. Much effort has been focused on whether LIGs are powered principally by starburst or AGN activity, although both types of activity are almost certainly present. The discussion has been framed around a scenario proposed by Sanders et al. (1988) in which a merger of gas-rich disk galaxies stimulates a massive nuclear starburst which in turn feeds a coalescing AGN core in the galaxy nucleus. As the AGN turns on, radiation pressure drives out the shroud of dust, revealing a nascent quasar. The goal must be not only to understand the dominant source of energy in LIGs, but to understand the relationship between starburst and AGN activity and other galaxy characteristics, and to place them into an evolutionary context. We have approached this question by studying a complete sample of LIGs defined by Condon et al. (1991; CHYT); this work has concentrated on VLBI observations which offer unique AGN/starburst diagnostics in one of the few wavelength regimes where the optical depths to the active regions may fall below unity.

In an 18-cm VLBI survey of Luminous Infrared Galaxies for compact, high$T_{b}$ emission commonly associated with AGN activity, Lonsdale, Smith, and Lonsdale (1993; Paper I) showed that milli-arcsecond scale emission, $T_{b}>>10^{7} \mathrm{~K}$, is common, perhaps universal in LIGs. Furthermore, the LIGs follow a common relationship between core radio power and bolometric luminosity with radio-quiet QSOs (Lonsdale, Smith \& Lonsdale 1995). This lends support to the interpretation of LIGs as dust-enshrouded AGN. On the other hand, a recent detailed analysis of our VLBI survey data, (Smith, Lonsdale \& Lonsdale 1998; Paper II) investigated a starburst origin for LIGs in which the compact, high- $T_{b}$ emission is produced by luminous radio supernovae (RSN). This analysis indicates that most, but not all, LIG VLBI-scale emission may be modelled with starburstgenerated RSN, provided the RSN are extremely luminous and, in most cases, clustered. These predictions were confirmed with the detection of luminous RSN in the nuclei of Arp 220, consistent with a starburst origin for the infrared luminosity (Smith et al. 1998), described in $\S 2$. The AGN view is shown in $\S 3$ where we present our images of Mrk 231 which clearly show an AGN core which ignited within the last $\sim 10^{6}$ years in the center of a starburst disk.

\section{Arp 220: An Intense Starburst}

Arp 220 (= IC $4553 / 4=$ UGC $9913=$ IRAS $15327+2340$ ) is the archetype LIG with $L_{f i r} \approx 10^{12} L_{\odot}$ at a distance of $76 \mathrm{Mpc}\left(H_{0}=75 \mathrm{~km} \mathrm{~s}^{-1} \mathrm{Mpc}^{-1}\right)$. In previous $18 \mathrm{~cm}$ radio studies we showed that about $3 \%$ of the $18 \mathrm{~cm}$ radio power originates from compact, high- $T_{b}$ regions associated with its merging nuclei (Lonsdale, Smith \& Lonsdale 1993). First epoch images (1994 November) of Arp 220 at $3 \times 8$ mas angular resolution (Smith et al. 1998), showed over a dozen unresolved sources, $S_{18 \mathrm{~cm}}=0.2-1.2 \mathrm{mJy}$, within a $0.2 \times 0.4^{\prime \prime}(75 \times 150 \mathrm{pc})$ region centered on the NW nucleus and at least two additional sources in the $\mathrm{SE}$ nucleus. We interpreted these compact sources as luminous radio supernovae and presented a simple starburst model for Arp 220 which has a star-formation rate of $50-100 M_{\odot} y r^{-1}$ and a luminous radio supernova rate, $\nu_{L R S N} \sim 2 y r^{-1}$. 


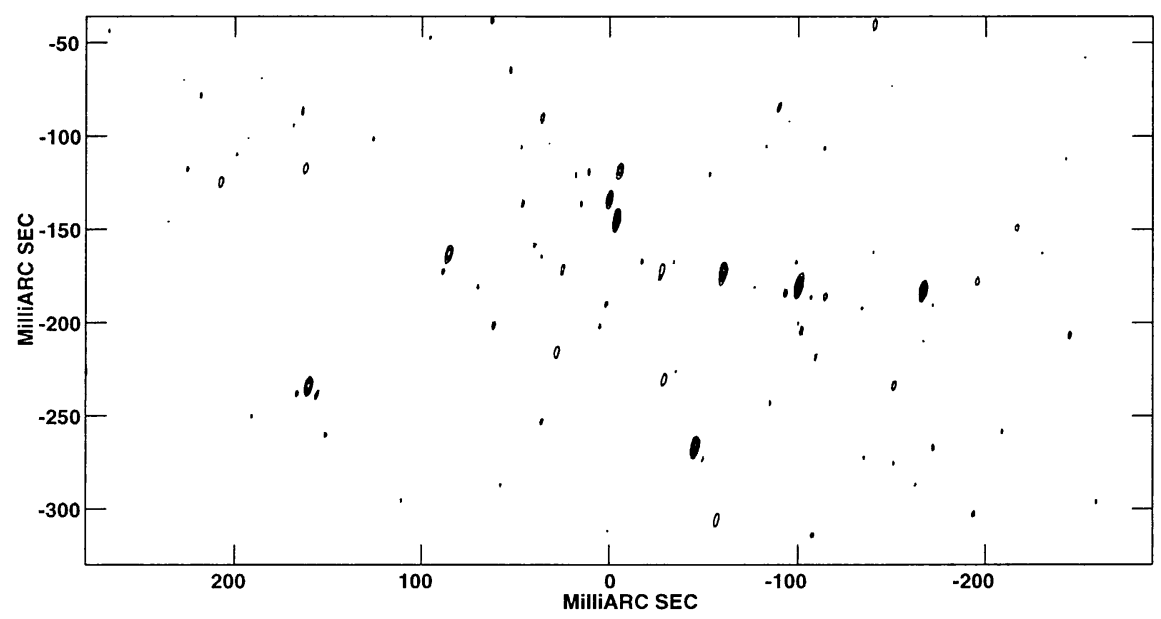

Figure 1. $18 \mathrm{~cm}$ VLBI image of the W Nucleus of Arp 220 from Smith, Lonsdale, Lonsdale \& Diamond (1998). Over a dozen unresolved sources are interpreted as luminous radio supernovae in an intense starburst.

Our second epoch observations, 3 years following the first epoch, show that most, but not all, of the compact radio sources have declined in brightness, but no new compact sources have appeared. Two of the compact sources are detected at $6 \mathrm{~cm}$; these sources have inverted $1.67-5.0 \mathrm{GHz}$ radio spectra, suggesting large optical depths, $\tau_{f f}(18 \mathrm{~cm}) \gtrsim 1.0-1.5$ and possibly young ages. These observations are consistent with the luminous RSN interpretation, but require a slower decline in the $18 \mathrm{~cm}$ radio light-curve with a concommittantly lower luminous supernova rate, $\nu_{L R S N} \lesssim 0.3 \mathrm{yr}^{-1}$, consistent with the lack of new RSN. If Arp 220 is powered by an enormous starburst, then only a fraction of its supernovae are luminous RSN, either because luminous RSN only occur in the most extreme environments, or because only very massive stars $\left(M \gtrsim 20 M_{\odot}\right)$ produce luminous RSN. We believe that it is likely that we are seeing only the upper part of the RSN luminosity function, formed in the densest molecular regions of the compact nuclear starburst.

\subsection{Compact $\mathrm{OH}$ Maser Emission}

VLBI spectroscopic studies have shown that the $1667 \mathrm{MHz}$ OH maser emission from Arp 220 is concentrated in four pc-scale sources, two in each of the nuclei (Lonsdale et al. 1998). Other LIGs (e.g. IIIZw35 \& IRAS17208-0014, Diamond et al. 1998) show similar compact maser emission. These compact maser sources exhibit only the $\mathrm{OH} 1667 \mathrm{MHz}$ feature, but may account for up to $2 / 3$ of the $1667 \mathrm{MHz}$ luminosity. The maser characteristics may be summarized as follows:

1. Compact maser dimensions are of order $R \sim$ a few pc. 
2. The masers are high gain, with amplification factors of order $10^{2}-10^{3}$, and are almost certainly highly saturated.

3. The compact masers do not subtend sufficient solid angle for the infrared radiation to produce the pumping; they must be collisionally pumped, perhaps by shocks.

4. The compact $\mathrm{OH}$ masers exhibit substantial velocity gradients, with $\frac{\Delta v}{\Delta r} \sim$ $30 \mathrm{~km} \mathrm{~s}^{-1} \mathrm{pc}^{-1}$. If interpreted as rotation these gradients imply mass concentrations in excess of $10^{6} M_{\odot}$.

These characteristcis are very different from the classical extended megamasers described by Baan (this volume). We speculate that these compact masers may be related to the onset of AGN activity.

\section{Mrk 231: A Nascent Quasar Emerging from A Starburst}

Mrk 231 (=UGC 5058; $D=173 \mathrm{Mpc}$ ) has long been recognized as a remarkable galaxy: it is the most luminous galaxy in the local $(z \lesssim 0.1)$ Universe with properties that place it among classical AGN and also infrared galaxies. The remarkable properties of Mrk 231 are summarized by Weedman (this volume).

\subsection{Mrk 231 as a QSO}

Mrk 231 has long been considered an infrared quasar - the luminosity of Mrk $231, L_{b o l} \gtrsim 10^{46} \mathrm{erg} \mathrm{s}^{-1}$, is comparable to other low-redshift radio-quiet QSOs and it falls in the midst of the radio power-luminosity relation for QSOs (and LIGs) constructed by Lonsdale, Smith \& Lonsdale (1995). Mrk 231 shows a strong, broad-emission-line spectrum with strong Fell emission and highly reddened line and continuum $\left(A_{V} \approx 2\right.$; Boksenberg et al. 1977). Mrk 231 is reported to be variable at both optical (Hamilton \& Keel 1987) and radio wavelengths (McCutcheon \& Gregory 1978). Mrk 231 has three broad-absorption-line systems: $v_{e j} \approx 4700 \mathrm{~km} / \mathrm{s}, v_{e j} \approx 6000 \mathrm{~km} / \mathrm{s}, v_{e j} \approx 8000 \mathrm{~km} / \mathrm{s}$ (Adams \& Weedman 1972 , Rudy, Foltz \& Stocke 1985). The $v_{e j} \approx 8000 \mathrm{~km} / \mathrm{s}$ absorption-line system is variable on timescales of order 2-3 yrs (Boroson et al. 1991). Mrk 231 is underluminous in x-rays (0.1-4.5kev; Rush et al. 1996) and soft $\gamma$-rays (Dermer et al. 1997) a characteristic it shares with other BAL objects, but it shows an AGN-like hard x-ray spectrum (Turner 1998).

\subsection{Mrk 231 as a Luminous Infrared Galaxy}

With an infrared luminosity, $\log L_{F I R}=12.35\left(L_{\odot}\right)$, Mrk 231 is the most luminous infrared galaxy in the complete sample of CHYT. Like other well-studied LIGs, the system is rich in molecular gas, $\log M_{\mathrm{H}_{2}} \approx 10.2\left(M_{\odot}\right)$ (Solomon et al. 1997 ) and it is one of the original $\mathrm{OH}$ megamaser systems (Baan 1985). Furthermore, Mrk 231 shows tidal tails and other evidence for merger or disturbance in the host galaxy, which is itself a luminous system with evidence for rapid star formation (Hamilton \& Keel 1987, Sanders et al. 1988).

Bryant \& Scoville (1996) have interpreted the CO distribution as a molecular disk containing an estimated $M_{H_{2}} \approx 3 \times 10^{9} M_{\odot}$ within $1^{\prime \prime}(=840 \mathrm{pc})$ with 


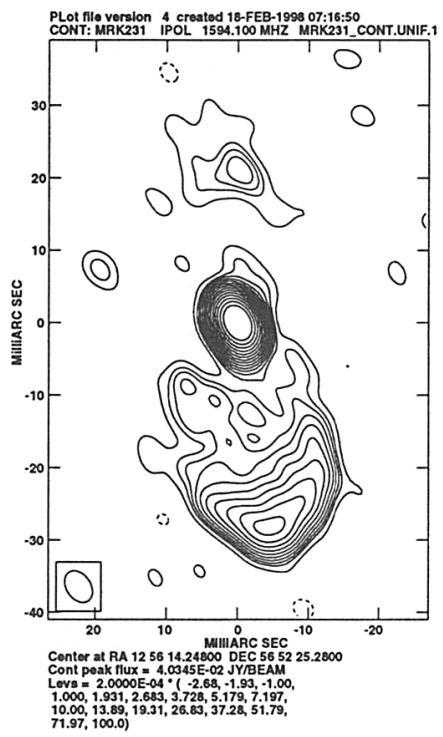

Figure 2. $18 \mathrm{~cm}$ VLBI image of the central 100pc of Mrk 231.

a scale height, $h \approx 30 p c$. Carilli, Wrobel and Ulvestad (1998) have detected HI absorption against the inner disk $(r \lesssim 160 p c)$. The HI observations suggest $N_{H I} \approx 6 \times 10^{22}\left(T_{s} / 1000 \mathrm{~K}\right) \mathrm{cm}^{-2},\left\langle n_{H I}>\approx 250\left(T_{s} / 1000 \mathrm{~K}\right) \mathrm{cm}^{-3}\right.$, and $M_{H I} \approx 2 \times 10^{7}\left(T_{s} / 1000\right) M_{\odot}$. Our $\mathrm{OH}$ maser observations show only extended $1667 \mathrm{MHz}$ emission within the central $100 \mathrm{pc}$. The radio continuum emission from the disk suggests that there remains significant star-formation activity, $\dot{m} \sim 60-100 M_{\odot} \mathrm{yr}^{-1}$.

\subsection{The Compact AGN Source at the Center}

Our image of the center of Mrk 231 shows a compact asymmetric source classified as a Compact Symmetric Object (CSO; Readhead et al. 1996b). The CSOs are believed to be young objects (age $\lesssim 10^{4} \mathrm{yr}$ ) which will evolve into FRII radio sources. Our analysis supports this view for Mrk 231. Simple optically-thin synchrotron modelling of the dominant (southern) radio lobe, which is almost certainly the working surface of a nuclear sub-relativistic jet upon the ambient medium, produces an estimate of the pressure in particles and field, $P_{\text {rel }} \approx$ $7 \times 10^{-7} d y n \mathrm{~cm}^{-2}$. Equating this pressure to the ram pressure, $P_{\text {ram }} \sim \rho v^{2}$, provides an estimate of the advance speed of the south lobe, provided we have an estimate of the ambient density. The mean molecular density in the starforming disk is estimated to be of order $n \sim 250 \mathrm{~cm}^{-3}$. A stronger limit on the density of the medium surrounding the south lobe comes from our map of the spectral index distribution, and by inference the free-free optical depth, around the working surface, $\rho \lesssim 2 \times 10^{-19} \mathrm{~g} \mathrm{~cm}^{-3}$. This results in a lower limit, $v_{a d v} \gtrsim 10^{-4} c$, with a resulting upper limit on the timescale for the onset of 
activity, $\tau_{A G N} \lesssim 10^{6} \mathrm{yr}$. Mrk 231 is a young quasar; the estimate of its age is substantially less than the inferred age of the starburst, $\tau_{* B} \sim 10^{8} \mathrm{yr}$.

\section{Summary}

The VLBI characteristics of many Luminous Infrared Galaxies, like Arp 220, may be explained by starburst-generated luminous RSN, but some LIGs, like Mrk 231, must harbor AGN cores. Compact OH megamaser emission is frequently present in LIGs and may be related to AGN activity. The scenario of Sanders et al. (1988) from merger - luminous starburst - QSO remains an attractive picture for the interpretation of LIGs.

\section{References}

Adams, T., \& Weedman, D. 1972, Ap.J., , 173, L109.

Baan, W. 1985, Nature, 315, 26. Ap.J., , 419, 553.

Boksenberg, A., et al. 1977, M.N.R.A.S., , 178, 451.

Boroson, T., Meyers, K., Morris, S., \& Persson, S. E. 1991, Ap.J., , 370, L19.

Bryant, P. \& Scoville, N. 1996, Ap.J., , 457, 678.

Carilli, C., Wrobel, J., \& Ulvestad, J. 1998, Astron. J., , 115, 928.

Condon, J., Huang, Z.-P., Yin, Q., \& Thuan, T. 1991, Ap.J., , 378, 65.

Dermer, C., et al. 1997, Ap.J., , 484, L121.

Diamond, P., Lonsdale, C., Lonsdale, C. \& Smith, H. E. 1998, Ap.J., , in press.

Hamilton, D. \& Keel, W. 1987, Ap.J., , 321, 211.

Lonsdale, C., Diamond, P., Lonsdale, C. \& Smith, H. E. 1998, Ap.J., , 493, L13.

Lonsdale, C., Smith, H. E., \& Lonsdale, C. 1995, Ap.J., , 438, 632.

Lonsdale, C., Smith, H. E., \& Lonsdale, C. 1993, Ap.J., , 405, L9 (Paper I).

McCutcheon, W. \& Gregory, P. 1978, Astron. J., , 83, 566.

Readhead, A., Taylor, G., Pearson, T., \& Wilkinson, P. 1996, Ap.J., , 460, 634.

Rudy, R., Foltz, C. \& Stocke, J. 1985, Ap.J., , 288, 531.

Rush, B., Malkan, M., Fink, H., \& Voges, W. 1996, Ap.J., , 471, 190.

Sanders, D. B. \& Mirabel, I. F. 1996, ARA\&A, 34, 749.

Sanders, D., et al. 1988, Ap.J., , 325, 74.

Smith, H. E., Lonsdale, C., \& Lonsdale, C. 1998, Ap.J., , 492, 137 (Paper II).

Smith, H. E., Lonsdale, C., Lonsdale, C. \& Diamond, P. 1998, Ap.J., , 493, L17.

Solomon, P., Downes, D., Radford, S., \& Barrett, J. 1997, Ap.J., , 478, 144.

Turner, T. J. 1998, Ap.J., , in press. 